\title{
Who Comes First? The Role of the Prefrontal and Parietal Cortex in Cognitive Control
}

\author{
Marcel Brass ${ }^{1}$, Markus Ullsperger ${ }^{1}$, Thomas R. Knoesche ${ }^{1}$, \\ D. Yves von Cramon ${ }^{1}$, and Natalie A. Phillips ${ }^{2}$
}

\begin{abstract}
Cognitive control processes enable us to adjust our behavior to changing environmental demands. Although neuropsychological studies suggest that the critical cortical region for cognitive control is the prefrontal cortex, neuroimaging studies have emphasized the interplay of prefrontal and parietal cortices. This raises the fundamental question about the different contributions of prefrontal and parietal areas in cognitive control. It was assumed that the prefrontal cortex biases processing in posterior brain regions. This assumption leads to the hypothesis that neural activity in the prefrontal cortex should precede parietal activity in cognitive control. The present study tested this assumption by combining results from functional magnetic resonance imaging (fMRI) providing high spatial resolution and event-related potentials (ERPs) to gain high temporal resolution. We
\end{abstract}

\section{INTRODUCTION}

Everyday life requires flexible and ongoing adjustment to different task situations. We can easily switch from one cognitive or motor task to the next with seemingly minimal effort. However, from cognitive psychology, we know that this flexibility requires higher-order cognitive control processes (Meiran, 1996; Monsell, 1996). One fundamental question in cognitive neuroscience relates to the neural mechanisms involved in these processes. From the neuropsychological perspective, the answer to this question seems to be relatively clear: Patients with prefrontal lesions have problems in situations which require the flexible adjustment to different task demands (Owen et al., 1993; Milner, 1963), suggesting that cognitive flexibility is critically reliant on the prefrontal cortex. The neuroimaging literature provides a less straightforward answer. In recent years, a number of imaging studies have investigated cognitive control processes using a wide range of paradigms (Brass \& von

\footnotetext{
${ }^{1}$ Max Planck Institute for Human Cognitive and Brain Sciences, Leipzig, Germany, ${ }^{2}$ Concordia University, Montreal, Canada
}

collected ERP data using a modified task-switching paradigm. In this paradigm, a situation where the same task was indicated by two different cues was compared with a situation where two cues indicated different tasks. Only the latter condition required updating of the task set. Task-set updating was associated with a midline negative ERP deflection peaking around $470 \mathrm{msec}$. We placed dipoles in regions activated in a previous fMRI study that used the same paradigm (left inferior frontal junction, right inferior frontal gyrus, right parietal cortex) and fitted their directions and magnitudes to the ERP effect. The frontal dipoles contributed to the ERP effect earlier than the parietal dipole, providing support for the view that the prefrontal cortex is involved in updating of general task representations and biases relevant stimulus-response associations in the parietal cortex.
Cramon, 2002; Rubia et al., 2001; Banich et al., 2000; Dove, Pollmann, Schubert, Wiggins, \& von Cramon, 2000; MacDonald, Cohen, Stenger, \& Carter, 2000). In contrast to the neuropsychological literature, neuroimaging studies show that frontal and parietal brain regions are both involved in cognitive control, raising questions about the specific and unique contributions of prefrontal and parietal brain regions. Theories on the basis of single-unit recordings and lesion experiments in monkeys have assumed that the contribution of the prefrontal cortex is to bias processing in posterior brain regions (Miller \& Cohen, 2001; Tomita, Ohbayashi, Nakahara, Hasegawa, \& Mijashita, 1999). If this assumption holds true, one would expect prefrontal cortex activation to precede parietal activation during the implementation of cognitive control.

The aim of the present experiment was to test this assumption by combining results from a functional magnetic resonance imaging (fMRI) experiment which provides a high spatial resolution and event-related potentials (ERPs) to gain high temporal resolution. We used a task-switching paradigm (Monsell, 2003) to investigate the flexible adjustment to different task situations. The task-switching paradigm compared 
with other cognitive control paradigms allows one to separate task-related control processes from responserelated control processes by presenting a cue in advance of the task to be performed (Meiran, 1996). We have recently developed an experimental paradigm to investigate task preparation with fMRI (Brass \& von Cramon, 2004). In this paradigm, participants alternated between two tasks, namely, judging whether a number was odd or even (parity task) or judging whether the number was greater or less than 30 (magnitude task). Four arbitrary task cues were used, two of which signaled the magnitude task and two of which signaled the parity task. This cue-to-task mapping allows us to disentangle a switch of the cue from a task switch (Logan \& Bundesen, 2003; Mayr \& Kliegel, 2003). In most of the trials, two sequential task cues preceded the target and were both followed by a preparation interval (Figure 1). In addition, catch trials were presented in which the target appeared after the first cue. These catch trials ensured that participants paid attention to the first cue. By comparing a switch of cues and cue meaning (two different cues that indicate different tasks, meaning switch [MS]) to a switch of cues without a switch of cue meaning (two different cues that indicate the same tasks, meaning repetition $[\mathrm{MR}]$ ), we could investigate the updating of the task representation without confounding cue encoding. Although the cue changes in both conditions, the task set changes

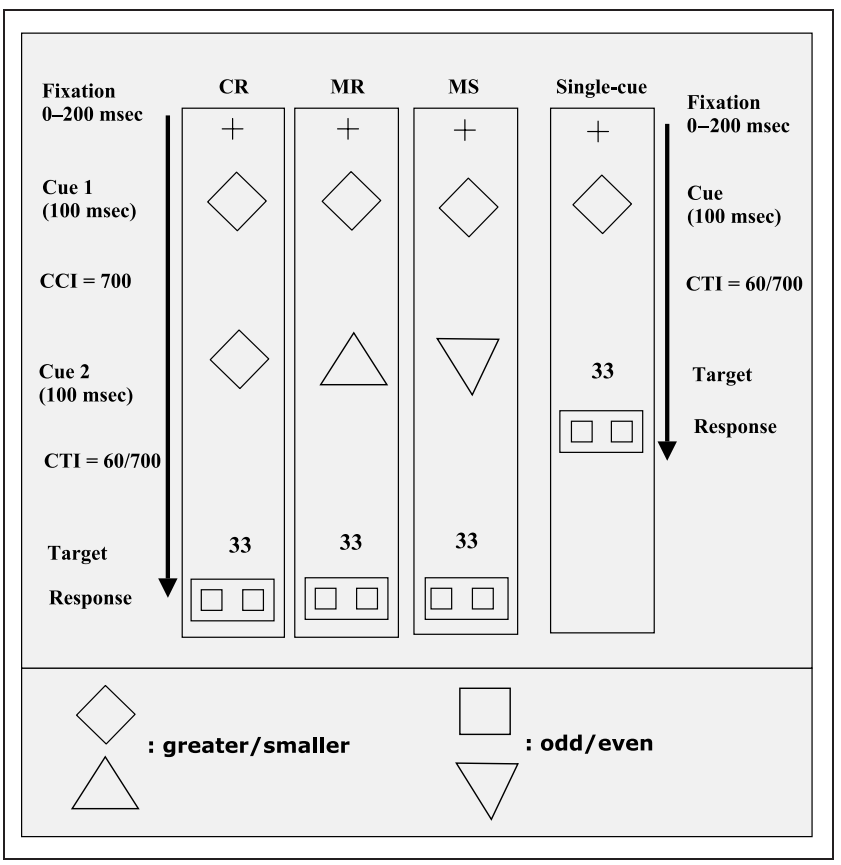

Figure 1. Trial structure and exact experimental timing. In the three double-cue conditions, two task cues were presented before the target was displayed. The cues were separated by a fixed CCI. In the single-cue condition, the target was presented after the first cue. The bottom part of the figure displays the cue-task mapping. Two different cues were assigned to each task. only in the MS condition. In addition to trial type, we manipulated the cue-target interval (CTI). In the short CTI, participants were required to respond $60 \mathrm{msec}$ after the relevant cue (the second cue in the three double-cue conditions and the first cue in the singlecue condition), whereas in the long CTI condition, they had $700 \mathrm{msec}$ to prepare. The CTI manipulation was introduced as an additional manipulation check to ensure that the first cue was indeed processed. If the double-cue conditions differ regarding the processes invoked, we would expect a reaction time (RT) difference in the short CTI condition, but not in the long CTI condition, because participants are not able to prepare the second cue in the short CTI before the target is presented. Hence, the experimental design consisted of the factors Trial type (MR, MS) and CTI (short, long). The single-cue condition was analyzed separately because of the different trial structure.

In a recent fMRI study, we showed that regions in the posterior fronto-lateral cortex and in the intraparietal sulcus (IPS) are related to the updating of the task (Brass \& von Cramon, 2004). By using the cortical foci from this fMRI experiment to model the spatial sources of ERP data collected with the identical paradigm, we investigated whether prefrontal cortex activation precedes the parietal cortex activation. If so, these data would strongly support the assumption of a hierarchical organization of prefrontal and parietal cortices in cognitive control.

\section{RESULTS}

\section{Behavioral Findings}

Figure 2 depicts the main behavioral findings. In the single-cue condition, RTs were faster when the CTI was long than when it was short. This effect of CTI was significant $\left(T_{18}=-4.79, p<.001\right)$, suggesting that the first cue was used to prepare the relevant task. A similar finding was present for error rates, however, it did not reach significance $\left(T_{18}=-1.64, p=.12\right)$.

We will now focus on the double-cue conditions in which the second cue was different from the first one. By comparing the MS and MR conditions, effects of differential task preparation processes can be extracted. RT and error rate data were subjected to repeatedmeasures ANOVAs with the factors Cue meaning (two levels: switch vs. repetition) and CTI (two levels: long vs. short), revealing main effects of Cue meaning [RT: $F(1,18)=19.82, p<.001$; error rates: $F(1,18)=6.02$, $p<.05]$ and CTI [RT: $F(1,18)=151.64, p<.001$; error rates: $F(1,18)=21.73, p<.001]$ and an interaction of these two factors [RT: $F(1,18)=20.35, p<.001$; error rates: $F(1,18)=7.41, p<.05]$. Subordinate ANOVAs performed separately for short and long CTIs confirmed that the effect of cue meaning was only present for the short CTI [RT: $F(1,18)=32.48, p<.001$; error rates: 


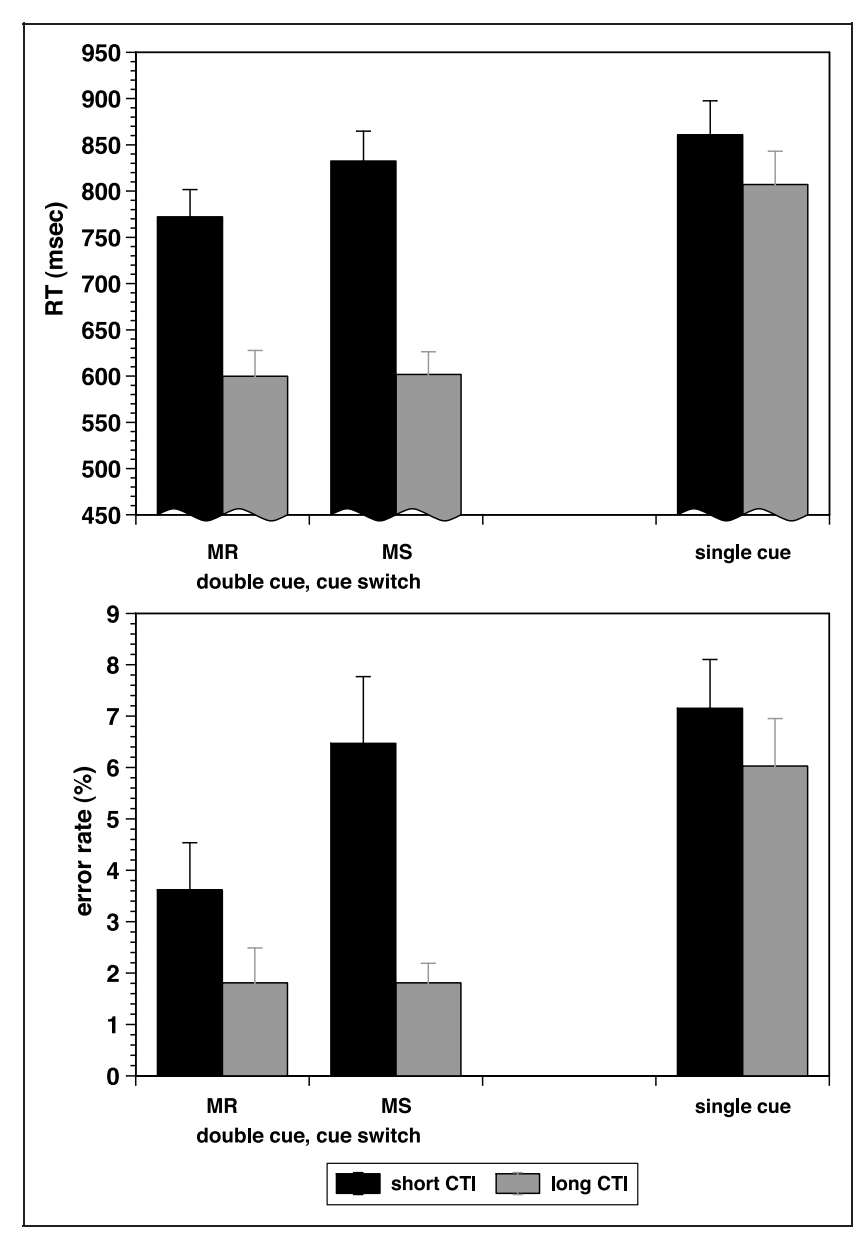

Figure 2. Behavioral findings. Response times (top) and error rates (bottom) in the different cueing conditions for short and long CTIs.

$F(1,18)=11.06, p<.005]$ and not for the long CTI $(p s>.8)$, suggesting that in these trials the interval between the second cue and the target onset was sufficiently long to implement the new task set in the MS condition. The effect of CTI was present in both the MR and MS conditions $(p s<.001)$.

When comparing the overall RT of the single-cue condition and the double-cue condition for the short CTI, an RT difference of about $200 \mathrm{msec}$ was found. We assume that this difference is due to the fact that participants did not always use the first cue to prepare the task. This is in accordance with the finding of DeJong, Berendsen, and Cools (1999) who could show that participants sometimes postpone task preparation until the target appears.

\section{ERP Findings}

The grand-average ERP waveforms for the conditions MR and MS, as well as the difference waveform (MS - MR) locked to the second cue, are depicted in Figure 3. As can be seen, the ERPs begin to differ around $400 \mathrm{msec}$ after the cue. In particular, in the MS condition, a negative-going deflection is visible peaking around $470 \mathrm{msec}$. It seems superimposed on a more sustained positivity present in both conditions and is much more prominent than in the MR condition. In both conditions, a negative-going slow wave (presumably a contingent negative variation $[\mathrm{CNV}]$ ) can be seen in the last 200 msec before target onset.

These observations were confirmed by statistical analyses. The ERP in the MS condition was significantly more negative than in the MR condition in the time range from 400 to $520 \mathrm{msec}$ in the right anterior, right central, and midline central regions of interest, and from 440 to 520 msec additionally in the midline anterior, left central, an left posterior regions. In the time range between 600 and $800 \mathrm{msec}$, the ERP for MS was more positive than for MR (in posterior regions starting at 600; in central regions starting at $640 \mathrm{msec}$, and in anterior regions starting at $680 \mathrm{msec}$ ), reflecting that the $\mathrm{CNV}$ had an earlier onset in the MR condition. ${ }^{1}$

\section{Dipole Modeling}

The dipole model accounted for $74 \%$ of the measurement variance. This leaves a rather large portion of the signal unexplained. However, the signal-to-noise ratio over all channels and time steps in the time window of the significant negative ERP effect of the grand average with respect to the baseline interval was just 4.4. This means that under the assumption that the noise in the analysis time interval is the same as in the baseline interval, only $81 \%$ of the variance of the observed signal stems from brain activity. This accounts for most of the unexplained variance (19\% out of $26 \%$ ). In other words, the spatio-temporal dipole fit of the directions and magnitudes of the dipoles yields a model explaining 91\% of the available variance, which is not attributed to noise. ${ }^{2}$ The directions of the computed dipoles are shown in Figure 4A. For the left fronto-lateral cortex and the right IPS, tangential dipoles have been found, indicating sulcal activity. For the right inferior frontal gyrus, a radial source was reconstructed, representing gyral activity. The time courses of the model are depicted in Figure 4B, suggesting that the frontal areas are particularly active during the early part of the investigated time window, whereas the IPS comes into play later. A repeated-measures ANOVA computed from those time steps, where the correlation between data and model was better than .9 (see Figure 4B), revealed a significant main effect for the factor Dipole $[F(2,21)=$ $3.27, p=.04]$ and a significant interaction between the factors Dipole and Window $[F(2,42)=3.51, p=.03]$. Follow-up pairwise $t$ tests revealed a significant difference between the two time windows for all three dipoles (see Figure 5). Thus, the statistics confirms the above-stated result that the frontal sources are more active in the early part of the N400 time window 
Figure 3. (A) Grand mean ERP waveforms for MS and MR for the epoch after onset of the second cue $(t=0)$ in the long CTI condition. (B) Difference wave resulting from subtracting cue switch from MS. Shaded bars indicate a significant difference of the difference wave from zero. (C) Topographical scalp distribution of the negative ERP difference in the time range $400-520 \mathrm{msec}$.

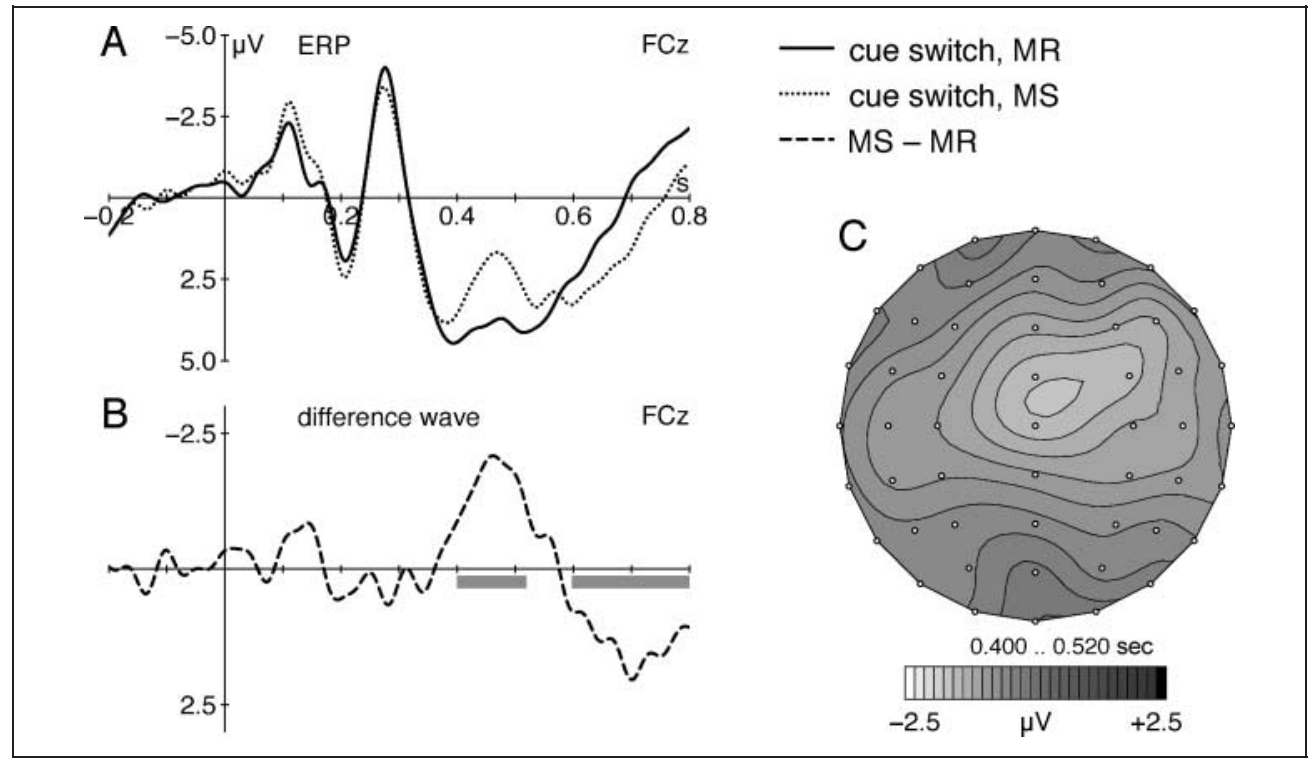

and the parietal source is stronger in the late part. Regarding the temporal characteristics of the left and right frontal cortex, the dipole analysis does not allow more specific conclusions.

\section{DISCUSSION}

By combining results from an fMRI experiment with ERP data, we show that prefrontal activity precedes parietal activity in cognitive control processes. These results provide strong evidence for the assumption that the parietal and prefrontal cortices serve different functions in cognitive control as was previously suggested in the context of research with nonhuman primates (Miller \& Cohen, 2001; Tomita et al., 1999). We assume that the prefrontal cortex provides an abstract task representation which is then further specified in the IPS.

\section{ERP Correlates of Task Updating}

Contrasting the condition in which participants were required to update the task representation with the condition in which only the cue but not the cue meaning changed revealed a negativity with a frontocentral maximum that peaked about $470 \mathrm{msec}$ after the presentation of the task cue. To our knowledge, this is the first ERP evidence for task updating when cue encoding was controlled. Considering that this negativity is associated with processing of a task representation that is incongruent with the task prepared after the first cue, it seems to share features with the N400, a compo-
Figure 4. (A) Dipoles for the negative ERP effect (400-500 msec). The positions were taken from Brass and von Cramon (2004), whereas the directions were obtained from the grand average of the task-switch effect in the ERP data by nonlinear fitting. Upper row: left inferior frontal junction (IFJ). Middle row: right inferior frontal gyrus (IFG). Lower row: right intraparietal sulcus (IPS). (B) Top: Time courses of the dipoles fitted to the grand average of the N400. Bottom: Time course of correlation of dipole model with grand-average data.

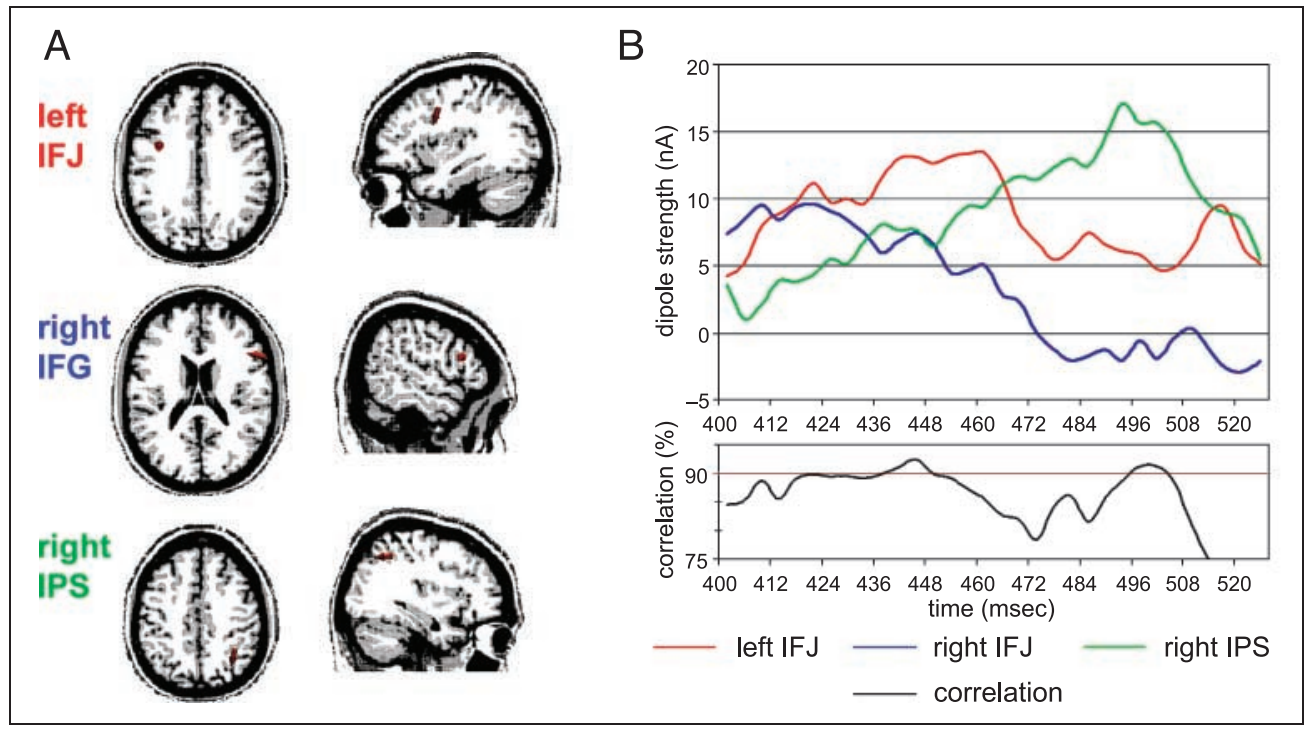




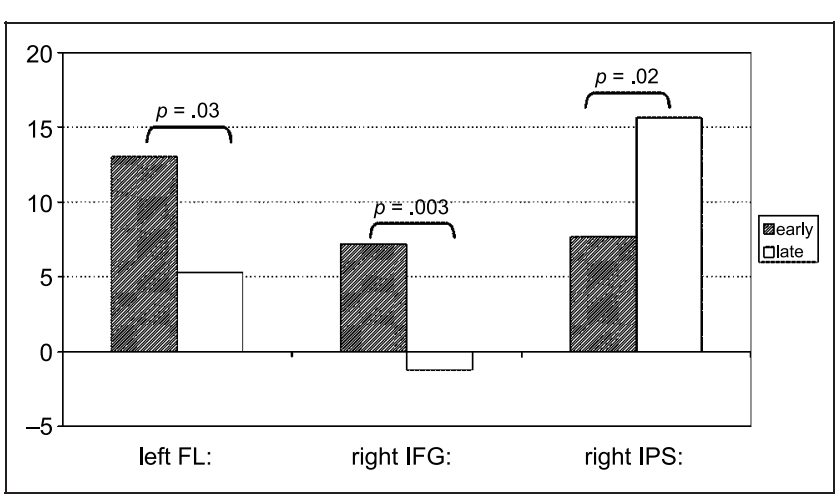

Figure 5. Dipole strength, averaged over subjects and time steps with a correlation between model and grand-average data greater than .9 in the early and late half of the N400 time window. The $p$ values refer to pairwise two-tailed $t$ tests.

nent first described in the language domain (Kutas \& Hillyard, 1980). N400-like deflections have been described for a number of occasions, in which the meaning of the stimulus violates the semantic context, and the occurrence of these deflections seems to be independent of the modality of either the context or the violating stimulus (Federmeier, Kluender, \& Kutas, 2003; Hamm, Johnson, \& Kirk, 2002). The amplitude of the N400 is assumed to reflect the amount of processing needed to integrate the stimulus into the given context. In the present experiment, the N400-like negativity is most pronounced when the task meaning indicated by the second cue is different from the task prepared on the first cue. In analogy to the N400 in other domains it could be assumed to reflect updating processes needed to integrate the required task representation into the context (i.e., task preparation). Interestingly, the scalp topography of the negativity seems to have a more frontal maximum than in the languagerelated N400 (which usually has a centro-parietal distribution). This is also reflected in the difference of our fMRI-based dipole model as compared to sourcelocalization findings in the language domain that suggested peri-sylvian sources (D'Arcy, Connolly, Service, Hawco, \& Houlihan, 2004; Helenius, Salmelin, Service, \& Connolly, 1998; Simos, Basile, \& Papanicolaou, 1997).

There are several neurophysiological studies which have investigated ERP components in task switching (Karayanidis, Coltheart, Michie, \& Murphy, 2003; Wylie, Javitt, \& Foxe, 2003; Rushworth, Passingham, \& Nobre, 2002) and variants of the Wisconsin Card Sorting (WCST) (Barcelo, 2003; Barcelo, Perianez, \& Knight, 2002). Interestingly, these studies have reported a positivity in the preparation interval (Karayanidis et al., 2003; Barcelo et al., 2002; Rushworth et al., 2002). It should be noted that we also found a more sustained P300-like positivity (from about 300 to $600 \mathrm{msec}$ ) for both conditions on which the differentially modulated negative deflection was superimposed. However, previ- ous studies are not directly comparable to the present study because they differed with respect to crucial experimental variables. In WCST studies and the study of Rushworth et al. (2002), the switch operation was embedded in a sequence of repetition trials (Barcelo, 2003; Barcelo et al., 2002; Rushworth et al., 2002). In these studies, the ERP modulation might have signaled the relative novelty of the switch operation compared with the repetition of the trial. This is exactly the interpretation favored by Barcelo et al. (2002), arguing that the P300, which was usually found in the context of bottom-up processing of novel nontargets, reflects some kind of top-down modulation in the context of set switching.

In the study of Karayanidis et al. (2003) and Wylie et al. (2003), switch and repetition trials were presented in close alternation. However, their study differed in another crucial aspect from the present experiment. They used a so-called alternating runs paradigm (Rogers \& Monsell, 1995). In this paradigm, the tasks are presented in a predictable order. This allows the participant to predict the identity of the upcoming task on the basis of the task sequence. In this paradigm, it is very difficult to determine when the preparation of the next trial starts. Interestingly, in the study of Karayanidis et al. (2003), a tendency for an N400-like component similar to the negativity reported here could be observed in the data (see Figure 7, p. 342, response-stimulus interval $1200 \mathrm{msec}$ ). However, this difference wave was not significant. This might be related to the fact that in alternating-runs paradigms the updating of the task representation is not temporally locked to a specific event and therefore a temporal jittering can be assumed.

Interestingly, studies on Stroop interference tasks using EEG have found a negative modulation when comparing incongruent and congruent trials (MarkelaLerenc et al., 2004; Liotti, Woldorff, Perez, \& Mayberg, 2000; West \& Alain, 1999). The study by Markela-Lerenc et al. (2004), for example, reported an N400-like component for which they fitted a dipole into the left lateral prefrontal cortex. It is very reasonable to assume that the updating of the relevant task representations is a crucial process involved in Stroop interference (Monsell, Taylor, \& Murphy, 2001). In the task-switching paradigm, this updating process is required because participants alternate between different task representations and therefore are not able to adjust to only one task set. In the Stroop task, this process is required because an irrelevant, but dominant, task set (reading the color of the word) permanently interferes with the relevant task set. Accordingly, fMRI activations found in the Stroop task and in the task-switching paradigm overlap to a high degree (Derrfuss, Brass, Neumann, \& von Cramon, 2005; Derrfuss, Brass, \& von Cramon, 2004). However, in the Stroop task, task-related and response-related processes are confounded. Accordingly, most ERP studies which tried source localization in the Stroop task found 
a source in the fronto-median cortex. We assume that this fronto-median source, which peaked after the frontolateral source (Markela-Lerenc et al., 2004), reflects response-related processes, namely, monitoring for response conflict (Yeung, Botvinick, \& Cohen, 2004; Ullsperger \& von Cramon, 2001). In the present task, response conflict is not a confounding factor, as we investigated the time period before target presentation in which no response tendencies could be built up, yet.

In the last $200 \mathrm{msec}$ before target onset, a positive difference between the MS and MR conditions was found. This difference was due to the delayed onset of the CNV preceding the target stimulus in the MS condition. This finding provides a hint that in this condition more time was needed for an additional process preceding the CNV, namely, integrating the new contextual information to activate the relevant task set. Interestingly, Goffaux, Sinai, Pushkar, and Phillips (submitted) observed a switch-related modulation of negative slowwave activity in cue-locked ERPs in a task-switching paradigm as well.

\section{A Hierarchical Model of Posterior Prefrontal and Intraparietal Cortex Function in Cognitive Control}

In recent years, a number of neuroimaging studies have investigated the neural mechanisms involved in cognitive control processes (e.g., Banich et al., 2000; Dove, Pollmann, Schubert, Wiggins, \& von Cramon, 2000; MacDonald et al., 2000). Most of these studies provided evidence that not only the prefrontal cortex but also areas in the intraparietal cortex play a crucial role for the control of our behavior. The classical view was that the parietal cortex is involved in response-related processes. In a recent fMRI study, we showed that activation in the IPS was independent from response-related processes (Brass \& von Cramon, 2002). This raises the question whether it is really possible to separate the contribution of the IPS and posterior prefrontal cortex in cognitive control. Recently, Tomita et al. (1999) could further clarify the role of the prefrontal cortex and posterior cortices in a memory task. Using a lesion approach in nonhuman primates, they could demonstrate that the prefrontal cortex biases processing in memory-related posterior cortices. A similar hierarchical organization could be assumed for the relation of the posterior prefrontal cortex and the IPS in cognitive control. One way to test such an assumption in human subjects is to experimentally manipulate potential processes related to the prefrontal and parietal cortex (e.g., Bunge, Hazeltine, Scanlon, Rosen, \& Gabrieli, 2002). In the present study, we used the high temporal resolution of EEG in combination with results from an fMRI study to temporally separate the contribution of the prefrontal cortex and the parietal cortex in cognitive control. Dipole modeling of the observed negative ERP effect suggests that its first part mostly resulted from activity in the lateral prefrontal cortex. Later the activity shifted to the parietal cortex. These findings strongly suggest that the lateral prefrontal cortex precedes the intraparietal cortex in cognitive control. A similar result was reported by Rushworth et al. (2002). The parietal cortex came into play before the task was presented. Even if the parietal areas are involved in preparationrelated processes (Ruge, Brass, Koch, Rubin, Meiran \& von Cramon, 2005; Brass \& von Cramon, 2002, 2004), the temporal order of prefrontal and parietal activation strongly suggests that both cortices provide task information on different levels of abstraction. In the lateral prefrontal cortex, the task representation is on a very abstract level. It specifies the task goal in general terms. Such a representation might be coded in a languagelike format. In the IPS, this abstract task representation is further specified. How close such a representation maps the actual task description is an open question, which has to be addressed in future research.

\section{METHODS}

\section{Participants}

Nineteen healthy volunteers ( 8 women) recruited at the University of Leipzig participated in this study. Their age ranged from 22 to 37 years (mean 25.6), they were all right-handed and had normal or corrected-to-normal vision. Written informed consent according to the Declaration of Helsinki was obtained prior to the study.

\section{Experimental Design}

In this study, we used a modified version of the paradigm introduced by Sudevan and Taylor (1987). Numbers between 20 and 40 (except 30) were presented on the computer screen. Participants had to perform two tasks: judging whether a number was smaller or greater than 30 (magnitude task) and judging whether the number was odd or even (parity task). Which task they had to execute on any given trial was signaled by a task cue presented as a frame surrounding the target number. In the double-cue conditions, participants received two task cues before the actual task was presented. These task cues could indicate the same (e.g., magnitude, magnitude) or a different task (magnitude, parity). Each experimental trial began with a fixation cross which was presented for $200 \mathrm{msec}$. The first task cue was then presented for $100 \mathrm{msec}$, followed 700 msec later by the second cue (i.e., a fixed cue-cue interval $[\mathrm{CCI}])$. After the presentation of the second cue for $100 \mathrm{msec}$, there was a CTI of 60 or $700 \mathrm{msec}$, after which the target was presented for $400 \mathrm{msec}$. Participants had $2000 \mathrm{msec}$ to respond to the target, after which the response window feedback (correct, incorrect) was displayed for $200 \mathrm{msec}$. In single-cue trials, the target was presented 60 or 
700 msec after the first and only cue. Two different task cues were assigned to each task (triangle and diamond to the parity task and square and inverted triangle to the magnitude task). This resulted in three double-cue conditions: a condition in which both the cue and the cue meaning were repeated (cuerepetition condition $[\mathrm{CR}]$ ), a condition in which the cue switched, but both cues indicated the same task (cue switch, MR condition), and a condition in which the cue switched to indicate the other task (cue switch, MS condition).

The experiment consisted of three blocks, with 320 trials each. There were 576 double-cue trials (192 CR/192 MR /192 MS) randomly intermixed with 384 singlecue trials. In $50 \%$ of each trial type, the CTI (time difference between onsets of last cue and target) was long (800 msec), in the other half of trials it was short $(160 \mathrm{msec})$. Trial types were presented in randomized order.

\section{ERP Data Collection and Analysis}

Participants were seated comfortably in a dimly lit, acoustically shielded chamber. The electroencephalogram (EEG) was recorded with $\mathrm{Ag} / \mathrm{AgCl}$ electrodes from 62 electrode sites (the extended 10-20 system) referenced to left mastoid and off-line re-referenced to the average voltage of both mastoids. Electrode impedance was kept below $5 \mathrm{k} \Omega$. The vertical electro-oculogram (EOG) was recorded from electrodes placed above and below the right eye. To monitor horizontal eye movements, the EOG was collected from electrodes placed on the outer canthus of the left and right eyes. EEG and EOG were recorded continuously with a low-pass filter of $70 \mathrm{~Hz}$ and $\mathrm{AD}$ converted with 22-bit resolution at a sampling rate of $250 \mathrm{~Hz}$.

As we were interested in task preparation, analysis focused on the interval between second cue and target onset in the double-cue condition with long CTIs. ${ }^{3}$ Therefore, signals in the epochs ranging from $200 \mathrm{msec}$ before to $800 \mathrm{msec}$ after the onset of the second cue were averaged. The average voltage in the $200 \mathrm{msec}$ preceding the cue onset served as a baseline. Prior to averaging, the EEG epochs were scanned for muscular and EOG artifacts. Whenever the standard deviation in a 200-msec interval exceeded $30 \mu \mathrm{V}$ in the EOG channels, the epoch was rejected.

For statistical analysis, electrode sites were pooled to form nine topographical regions (Gevins et al., 1996; Oken \& Chiappa, 1986). The following regions of interest were defined: left anterior (AF7, F5, F7, F3), midline anterior $(\mathrm{AFz}, \mathrm{Fz}, \mathrm{AF} 3, \mathrm{AF} 4)$, right anterior $(\mathrm{AF} 8, \mathrm{~F} 6, \mathrm{~F} 8$, F4), left central (FC5, T7, C5, C3), midline central (FCz, CPz, FC3, FC4), right central (FT8, T8, TP8, C6), left posterior (P5, TP7, CP5, CP3), midline posterior $(\mathrm{CPz}$, $\mathrm{Pz}, \mathrm{P} 3, \mathrm{P} 4)$, and right posterior (CP4, CP6, TP8, P6). We tested for the effects of cue meaning at these scalp regions over successive 40-msec time bins by means of $t$ tests. ERP differences were only considered significant, if they persisted for at least two consecutive time bins.

For the analysis of the spatio-temporal properties of the observed ERP effect, we made use of the superior spatial resolution of fMRI and the better temporal resolution of the ERP. In the fMRI study using the same paradigm as in the present work, Brass et al. (2004) identified three regions when contrasting the MS and MR conditions: left fronto-lateral cortex, right inferior frontal gyrus, and right IPS (see Figure 4A). In order to obtain information on the temporal evolvement of the activity in these areas, we placed a dipole in each of them and fitted their directions and magnitudes to the grand average of the difference between conditions MS and MR in the time range of the significant ERP effect (400-520 msec). We employed the so-called fixed dipoles model (Scherg \& Berg, 1991), assuming the directions of the dipoles to be constant over the entire time interval of analysis, whereas the magnitudes (or strengths) are allowed to assume different values for every time step. This resulted in a direction vector and a magnitude time course for each of the three dipoles. The plausibility and significance of this result were then tested in three different ways. First, we compared the portion of the variance explained by the model to the signal-to-noise ratio of the data, computed as the ratio of variances in the analysis and the baseline time windows. The dipole model should explain a fair amount of the data variance after the noise variance has been subtracted, with some unexplained variance allowed to account for model mismatch errors. Second, the computed dipole directions were assessed for plausibility. Gyral activity, as in the inferior frontal gyrus, should be represented by a radially oriented dipole, whereas sulcal activity, as in the IPS, should be reflected by tangential directions. Third, the data of the individual subjects were projected linearly onto the dipole model and the obtained magnitudes were investigated for statistical significance by variance analysis. The analysis time window was divided into an early and a late subwindow ( 400 to $460 \mathrm{msec}$ and 464 to $520 \mathrm{msec}$ ). Within each subwindow, only those time steps were averaged, for which the dipole model correlated with the grand-average data with more than .90 (Figure 4B). These averages served as a dependent variable in a repeated-measures ANOVA over subjects with the factors Dipole (three levels) and Window (two levels). If the brain activity distributed significantly differently over the three activation sites in the early N400 time window than in the late one, an interaction between the two factors should be found.

\section{Acknowledgments}

Reprint requests should be sent to Marcel Brass, MPI for Human Cognitive and Brain Sciences, Stephanstr. 1A, D-04103 Leipzig, Germany, or via e-mail: brass@cbs.mpg.de. 


\section{Notes}

1. The time course of the CNV was investigated in detail at electrode $\mathrm{CPz}$. The onset of the $\mathrm{CNV}$ was determined by comparing the amplitude at each sample point between 540 and $800 \mathrm{msec}$ with the mean amplitude in the time window 520-550 msec (after the significant negative ERP effect) for each condition by $t$ tests. The first time point at which the difference reaches significance was considered the onset of the CNV. For MR, the onset was $572 \mathrm{msec}$ after cue onset, for MS it was 688 msec. To determine the slopes of the CNV, linear regression analyses were calculated for the ERPs of each participant for the time window between the onset and $800 \mathrm{msec}$. The mean slopes were $-0.033 \mu \mathrm{V} / \mathrm{msec}$ (SEM 0.004) for MR and $-0.035 \mu \mathrm{V} / \mathrm{msec}$ (SEM 0.007) for MS and were not different between both conditions $\left(T_{18}=.3, p=.77\right)$. These results suggest that both conditions are associated with a CNV of similar slope, which is delayed in the MS condition by about 116 msec.

The statistical approach of binwise $t$ tests yields the potential problem of false-positive findings as a result of multiple tests. Two reasons render this problem unlikely for the present findings. First, the significant effects lasted for at least three adjacent time bins. Second, at least three regions of interest showed the effect at the same time.

2. If the position constraint of the dipoles is released, they move only a short distance $(<6 \mathrm{~mm})$ and the explained variance changes only from $74 \%$ to $75 \%$. This suggests an (at least locally) optimal solution.

3. A full report on the ERP measures as a function of cue and CTI condition is forthcoming.

\section{REFERENCES}

Banich, M. T., Milham, M. P., Atchley, R. A., Cohen, N. J., Webb, A, Wszalek, T., Kramer, A. F., Liang, Z., Barad, V., Gullet, D., Shah, C., \& Brown, C. (2000). Prefrontal regions play a predominant role in imposing an attentional 'set': Evidence from fMRI. Brain Research, Cognitive Brain Research, 10, $1-9$.

Barcelo, F. (2003). The Madrid card sorting test (MCST): A task switching paradigm to study executive attention with event-related potentials. Brain Research Protocols, 11, 27-37.

Barcelo, F., Perianez, J. A., \& Knight, R. T. (2002). Think differently: A brain orienting response to task novelty. NeuroReport, 13, 1887-1892.

Brass, M., \& von Cramon, D. Y. (2002). The role of the frontal cortex in task preparation. Cerebral Cortex, 12, 908-914.

Brass, M., \& von Cramon, D. Y. (2004). Decomposing components of task preparation with functional magnetic resonance imaging. Journal of Cognitive Neuroscience, 16, 609-620.

Bunge, S. A., Hazeltine, E., Scanlon, M. D., Rosen, A. C., \& Gabrieli, J. D. E. (2002). Dissociable contribution of prefrontal and parietal cortices to response selection. Neuroimage, 17, 1562-1571.

D’Arcy, R. C., Connolly, J. F., Service, E., Hawco, C. S., \& Houlihan, M. E. (2004). Separating phonological and semantic processing in auditory sentence processing: A high-resolution event-related brain potential study. Human Brain Mapping, 22, 40-51.

De Jong, R., Berendsen, E., \& Cools, R. (1999). Goal neglect and inhibitory limitations: Dissociable causes of interference effects in conflict situations. Acta Psychologica, 101, 379-394.
Derrfuss, J., Brass, M., Neumann, J., \& von Cramon, D. Y. (2005). Involvement of the inferior frontal junction in cognitive control: Meta-analyses of switching and Stroop studies. Human Brain Mapping, 25, 22-34.

Derrfuss, J., Brass, M., \& von Cramon, D. Y. (2004). Cognitive control in the posterior frontolateral cortex: Evidence from common activations in task coordination, interference control and working memory. Neuroimage, 23, 604-612.

Dove, A., Pollmann, S., Schubert, T., Wiggins, C. J., \& von Cramon, D. Y. (2000). Prefrontal cortex activation in task switching: An event-related fMRI study. Cognitive Brain Research, 9, 103-109.

Federmeier, K. D., Kluender, R., \& Kutas, M. (2003). Aligning linguistic and brain views on language comprehension. In A. Zani \& A. M. Proverbio (Eds.), The cognitive electrophysiology of mind and brain (pp. 143-168). San Diego: Academic Press.

Gevins, A., Smith, M. E., Le, J., Leong, H., Bennett, J., Martin, N., McEvoy, L., Du, R., \& Whitfield, S. (1996). High resolution evoked potential imaging of the cortical dynamics of human working memory. Electroencephalography and Clinical Neurophysiology, 98, 327-348.

Goffaux, P., Sinai, M., Pushkar, D., \& Phillips, N. (submitted). Behavioral and electrophysiological measures of task switching during single and mixed-task conditions.

Hamm, J. P., Johnson, B. W., \& Kirk, I. J. (2002). Comparison of the N300 and N400 ERPs to picture stimuli in congruent and incongruent contexts. Clinical Neurophysiology, 113, 1339-1350.

Helenius, P., Salmelin, R., Service, E., \& Connolly, J. F. (1998). Distinct time courses of word and context comprehension in the left temporal cortex. Brain, 121, 1133-1142.

Karayanidis, F., Coltheart, M., Michie, P. T., \& Murphy, K. (2003). Electrophysiological correlates of anticipatory and poststimulus components of task switching.

Psychophysiology, 40, 329-348.

Kutas, M., \& Hillyard, S. A. (1980). Reading senseless sentences: Brain potentials reflect semantic incongruity. Science, 207, 203-205.

Liotti, M., Woldorff, M. G., Perez, R., \& Mayberg, H. S. (2000). An ERP study of the temporal course of the Stroop color-word interference effect. Neuropsychologia, 38, 701-711.

Logan, G. D., \& Bundesen, C. (2003). Clever homunculus: Is there an endogenous act of control in explicit task cuing procedures? Journal of Experimental Psychology: Human Perception and Performance, 29, 575-599.

MacDonald, A. W., III, Cohen, J. D., Stenger, V. A., \& Carter, C. S. (2000). Dissociating the role of the dorsolateral prefrontal and anterior cingulate cortex in cognitive control. Science, 288, 1835-1838.

Markela-Lerenc, J., Ille, N., Kaiser, S., Fiedler, P., Mundt, C., \& Weisbrod, M. (2004). Prefrontal-cingulate activation during executive control: Which comes first? Cognitive Brain Research, 18, 278-287.

Mayr, U., \& Kliegl, R. (2003). Differential effects of cue changes and task changes on task-set selection costs. Journal of Experimental Psychology: Learning, Memory, and Cognition, 29, 362-372.

Meiran, N. (1996). Reconfiguration of processing mode prior to task performance. Journal of Experimental Psychology: Learning, Memory, and Cognition, 22, 1124-1442.

Miller, E. K., \& Cohen, J. D. (2001). An integrative theory of prefrontal cortex function. Annual Review of Neuroscience, 24, 167-202.

Milner, B. (1963). Effects of different brain lesions on card sorting. Archives of Neurology, 9, 90-100. 
Monsell, S. (1996). Control of mental processes. In V. Bruce (Ed.), Mysteries of the mind: Tutorial essays on cognition (pp. 93-148). Hove, UK: Erlbaum.

Monsell, S. (2003). Task switching. Trends in Cognitive Sciences, 7, 134-140.

Monsell, S., Taylor, T. J., \& Murphy, K. (2001). Naming the color of a word: Is it responses or task sets that compete? Memory \& Cognition, 29, 137-151.

Oken, B. S., \& Chiappa, K. H. (1986). Statistical issues concerning computerized analysis of brainwave topography. Annals of Neurology, 19, 493-497.

Owen, A. M., Roberts, A. C., Hodges, J. R., Summers, B. A., Polkey, C. E., \& Robbins, T. W. (1993). Contrasting mechanisms of impaired attentional set-shifting in patients with frontal lobe damage or Parkinson's disease. Brain, 116, 1159-1175.

Rogers, R. D., \& Monsell, S. (1995). Costs of a predictable switch between simple cognitive tasks. Journal of Experimental Psychology, 124, 207-231.

Rubia, K., Russell, T., Overmeyer, S., Brammer, M. J., Bullmore, E. T., Sharma, T., Simmons, A., Williams, S. C., Giampietro, V., Andrew, C. M., \& Taylor, E. (2001). Mapping motor inhibition: Conjunctive brain activations across different versions of go/no-go and stop tasks. Neuroimage, 13, 250-261.

Ruge, H., Brass, M., Koch, I., Rubin, O., Meiran, N., \& von Cramon, D. Y. (2005). Reconciling advance preparation and stimulus-induced interference in explicitly cued task switching: Further evidence from fMRI. Neuropsychologia, 43, 340-355.
Rushworth, M. F., Passingham, R. E., \& Nobre, A. C. (2002). Components of switching intentional set. Journal of Cognitive Neuroscience, 14, 1139-1150.

Scherg, M., \& Berg, P. (1991). Use of prior knowledge in brain electromagnetic source analysis. Brain Topography, 4, $143-150$.

Simos, P. G., Basile, L. F., \& Papanicolaou, A. C. (1997). Source localization of the $\mathrm{N} 400$ response in a sentence-reading paradigm using evoked magnetic fields and magnetic resonance imaging. Brain Research, 762, 29-39.

Sudevan, P., \& Taylor, D. A. (1987). The cuing and priming of cognitive operations. Journal of Experimental Psychology: Human Perception and Performance, 13, 89-103.

Tomita, H., Ohbayashi, M., Nakahara, K., Hasegawa, I., \& Mijashita, Y. (1999). Top-down signals from prefrontal cortex in executive control of memory. Nature, 401, 699-703.

Ullsperger, M., \& von Cramon, D. Y. (2001). Subprocesses of performance monitoring: A dissociation of error processing and response competition revealed by event-related fMRI and ERPs. Neuroimage, 14, 1387-1401.

West, R., \& Alain, C. (1999). Event-related neural activity associated with the Stroop task. Cognitive Brain Research, 8, 157-164.

Wylie, G. R., Javitt, D. C., \& Foxe, J. J. (2003). Task switching: A high-density electrical mapping study. Neuroimage, 20, 2322-2342.

Yeung, N., Botvinick, M. M., \& Cohen, J. D. (2004). The neural basis of error detection: Conflict monitoring and the error-related negativity. Psychological Review, 111, 931-959. 